\title{
Traumatic Arteriovenous Fistula of Superficial Temporal Vessel: A Rare Case Report
}

\author{
Kebebe Bekele' \\ Habtamu Gezahegn (iD ${ }^{2}$ \\ 'Madda Walabu University Goba Referral \\ Hospital, School of Medicine, \\ Department of Surgery, Bale-Goba, \\ Ethiopia; ${ }^{2}$ Madda Walabu University \\ Goba Referral Hospital, School of \\ Medicine, Department of Medical \\ Physiology, Bale-Goba, Ethiopia
}

\begin{abstract}
Arteriovenous fistulae are abnormal direct communications between artery and venous system. It rarely occurs in superficial temporal vessel and is usually caused by trauma. Clinical manifestation includes pulsatile mass, headache, hemorrhage and tinnitus. Angiography is standard diagnostic modality and surgical removal of the pulsating mass with ligature of the feeding vessel is definitive treatment. The authors describe the case of a 35-year-old female patient who developed a pulsatile tortuous mass, extending from the left preauricular area to the left scalp after sustaining thorn injury (extremely rare penetrating injury). The treatment done was a complete surgical removal of the pulsatile mass and ligature of the feeding superficial temporal artery.
\end{abstract}

Keywords: arteriovenous fistulae, superficial temporal vessel, thorn injury, case report

\section{Introduction}

Arteriovenous fistulae is direct communication between feeding high flow arterial segment and the low flow venous drainage system without intervening capillaries, resulting in a pulsatile, tortuous dilated vein. Patient may present with various clinical manifestations, such as bruits, tinnitus, local pain, hemorrhage, and disfiguring lesion. ${ }^{1-3}$

Traumatic arteriovenous fistula is arteriovenous shunting secondary to blunt, penetrating and iatrogenic trauma of artery and vein running in parallel, but rarely occur in the superficial temporal artery.

Arteriovenous fistula of the superficial temporal vessel is a rare condition with incidence of $0.5 \%$ to $2.0 \% ; 75 \%$ of superficial temporal arteriovenous fistula is caused by blunt, penetrating or iatrogenic trauma.,

In $90 \%$ of the patients with scalp arteriovenous fistula, superficial temporal vessel was involved. Several methods, such as open surgical removal, ligation of the feeding trans-vascular embolization, and intra-lesion injection of sclerosant, have been used to treat these cases. ${ }^{1}$

As to the knowledge of authors, there were no eports of superficial temporal vessel fistula in Africa. Throughout the world, superficial temporal vessel caused by penetrating injury is extremely rare and there is no report of thorn injury causing this fistula. The objective of this case report is to describe a case of extremely rare traumatic arteriovenous fistula of the temporal superficial vessel caused by thorn injury (penetrating) treated surgically in Ethiopia. Therefore, this case report tells us thorn injury can cause arteriovenous fistula.
Correspondence: Kebebe Bekele P.O Box 302, Bale Goba, Ethiopia Tel +251933205222

Email kebebeb2I@gmail.com 


\section{Case Report}

A 35-year-old female patient from rural area of Ethiopia presented with left lateral and scalp swelling of 15 years duration after she initially sustained thorn injury over the left preauricular area. At that time she had history of mild bleeding and she improved. But later, after two months of trauma, a small swelling started at the site of thorn injury then gradually and progressively involved the left upper lateral face and scalp area. In addition to swelling she had bruit, headache and tinnitus in left ear. Otherwise, she had no history of other trauma, smoking, chronic medical disease or alcohol use. For the above complaint, the patient went to nearby health center from where she was referred to Madda walabu University Goba Referral Hospital, Southeast Ethiopia.

Upon physical examination she was conscious and healthy looking, with the following vital signs: blood pressure $120 / 70 \mathrm{~mm} \mathrm{Hg}$, pulse rate of 90 beats per minute, temperature of $36.4{ }^{\circ} \mathrm{C}$ and respiratory rate of 20 breaths per minute. She had pink conjunctiva, non-icteric sclera and wet tongue. There was significant dilated, tortuous, visible pulsatile vessel over the left preauricular and scalp area. Skin overlying dilated vessel was normal and upon palpation there was significant pulsation and bruit sound heard upon auscultation over pulsatile mass.

With the impression of traumatic superficial temporal vessel arteriovenous fistula, the patient was investigated with hemoglobin of $13.6 \mathrm{gm} / \mathrm{dl}$, white blood cell of 12,300 / L platelet was 160,000/microliter. Doppler ultrasound confirmed arteriovenous fistula of superficial temporal vessel. But CT angiography was not done due to absence of such a service in the area.

With same diagnosis, patient was operated and intraoperative finding was pulsatile dilated superficial vessel. Feeding superficial temporal vessel was ligated at level of left ear. The patient was followed for 2 months and swelling was resolved and there was no recurrence (Figure 1).

\section{Discussion}

The superficial temporal artery is vulnerable to trauma because of its superficial path over the temporal bone and proximity to cranial sutures and because the superficial temporal artery and superficial temporal vein run in parallel with each other over the temporal bone. In addition to trauma, it can occur spontaneously or be caused by surgical procedures. Traumatic superficial temporal vessel fistulae develop over the course of months or years after

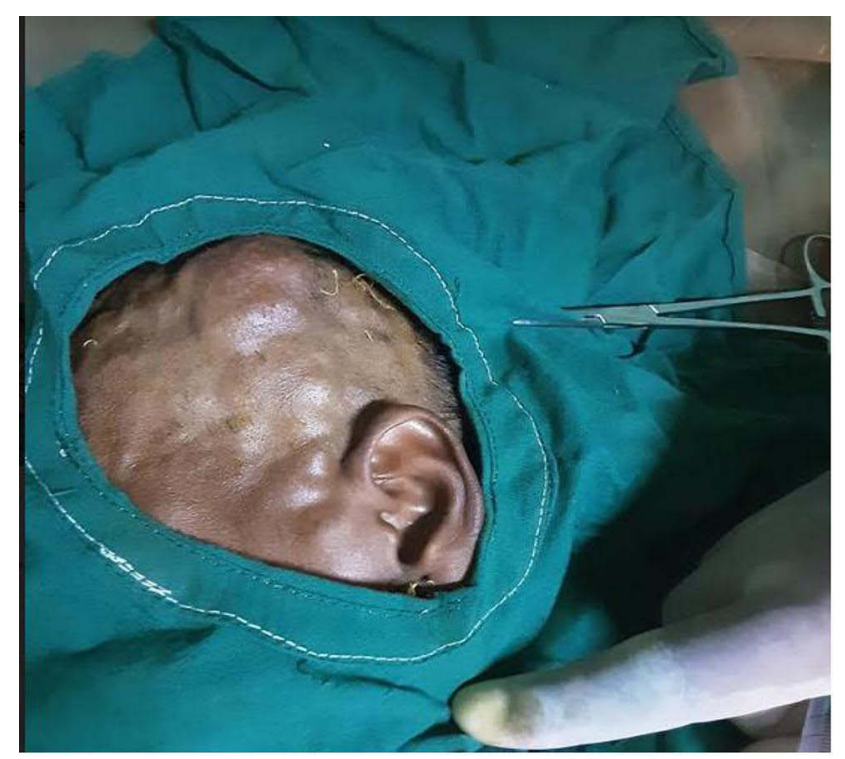

Figure I Picture showing pulsatile tortuous mass over left lateral face and scalp region in a 35 -year-old female patient.

the trauma. Spontaneous superficial temporal vessel fistulae may be present congenitally, even though the majority of cases remain asymptomatic until puberty. ${ }^{3}$

In general, the most common symptoms of arteriovenous fistula of the scalp are localized headache, a pulsatile subcutaneous mass and pulsatile tinnitus. Physical examination findings are: dilated and tortuous vessel, bruit on auscultation of the mass, pulsatile vibration on palpation, and Terrier's sign. ${ }^{5}$ One should consider superficial temporal arteriovenous fistula for possible cause of unilateral headache if patient felt bruit in ipsilateral ear.

Diagnosis of a traumatic fistula of the superficial temporal vessel is based on a history of trauma and a detailed physical examination. But angiography remains the gold standard investigation.

There are two possible mechanisms explaining pathophysiology of traumatic arteriovenous fistulae formation in the scalp. The first one is simultaneous laceration of the adjacent artery and vein. The second one, starts with the rupture of vasa vasorum in the artery wall, which stimulates endothelial proliferation from damaged vasa vasorum then forms numerous small vessels, leading to vascular connection between the two vessels. In our case, since thorn injury is a penetrating injury, it results in vascular fistula highly likely caused by the first mechanism.

Surgical excision, ligature of the feeding vessels, embolization and injection of sclerosing agents into the lesion are common treatment options, but surgical removal 
of the pulsating mass and ligature of the feeding vessel remains standard treatment.

The main advantage of embolization of this arteriovenous fistula is to reduce blood loss, but risk of recurrence is high, especially for complex fistula if done alone. ${ }^{1,3,6}$

\section{Consent}

Written consent was taken from our patient and recorded on patient consent form and also the patient provided written consent for the publication of her case, including images of her case, and hence we can provide if requested. However, institutional approval is not required to publish the case because we did not take any sample or do any procedure on the patient for research purposes. We only treated the patient and reported her case and the patient provided written consent for the publication of her case. Institutional approval is needed when samples are taken or procedures are done for research purposes.

\section{Disclosure}

The authors declare that there is no conflict of interest in this work.

\section{References}

1. Zheng F, Augustus Pitts H, Goldbrunner R, Krischek B. Traumatic arteriovenous fistula of the scalp in the left temporoparietal region with intra- and extracranial blood supply. Case Rep Vasc Med. 2016;2016:8671472. doi:10.1155/2016/8671472

2. Asai K, Tani S, Imai Y, Mineharu Y, Sakai N. Traumatic arteriovenous fistula of the superficial temporal artery. J Surg Case Rep. 2015;2015 (12):rjv156. doi:10.1093/jscr/rjv156

3. Camargo Júnior O, Abreu M, Abreu G, Gabriel SA, Silva IMMD. Traumatic arteriovenous fistula of the superficial temporal artery. J Vasc Bras. 2014;13(1):39-42. doi:10.1590/jvb.2014.008

4. Moran AM, Aleman TS, Gausas RE, Fogt F. Traumatic arteriovenous fistula of the superficial temporal artery: a histopathologic report. Ophthalmic Plast Reconstr Surg. 2013;29(5):e126-8. doi:10.1097/ IOP.0b013e31827ab9ef

5. Biegaj E, Rutkowska-Zimirska J, Radzymińska-Maliszewska M, Zaremba A, Pniewski J. Arteriovenous fistula of superficial temporal vessels. Folia Morphol. 2019;78(4):879-882. doi:10.5603/FM. a2019.0016

6. Yang M, Pan L, Cai M-J, et al. Spontaneous arteriovenous fistula of the superficial temporal artery: diagnosis and treatment. Clin Neurol Neurosurg. 2014;123:18-24. doi:10.1016/j.clineuro.2014.05.001
International Medical Case Reports Journal

\section{Publish your work in this journal}

The International Medical Case Reports Journal is an international, peer-reviewed open-access journal publishing original case reports from all medical specialties. Previously unpublished medical posters are also accepted relating to any area of clinical or preclinical science. Submissions should not normally exceed 2,000 words or 4

\section{Dovepress}

published pages including figures, diagrams and references. The manuscript management system is completely online and includes a very quick and fair peer-review system, which is all easy to use. Visit http://www.dovepress.com/testimonials.php to read real quotes from published authors. 